\title{
Fraunhofer
}

Fraunhofer Centre for Applied Photonics

\section{A Broadly Tunable Ultrafast Diode-Pumped Ti:sapphire Laser}

Jamie C. E. Coyle ${ }^{1,2}$, Alan J. Kemp², John-Mark Hopkins ${ }^{1}$ and Alexander A. Lagatsky ${ }^{1}$

1. Fraunhofer Centre for Applied Photonics, Fraunhofer UK Research Ltd, 99 George Street, Glasgow, G1 1RD, UK.

2. Institute of Photonics, Dept. of Physics, University of Strathclyde, 99 George Street, Glasgow, G1 1RD, UK.

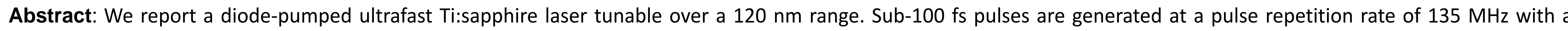
maximum average output power of $430 \mathrm{~mW}$

\begin{tabular}{|c|c|c|c|c|c|c|c|c|}
\hline \multicolumn{8}{|c|}{ Ultrafast diode-pumped Ti:sapphire lasers } & \multirow{6}{*}{$\begin{array}{l}\text { Mode-locking mechanismS } \\
\text { Here we report to the best of our knowledge the first broadly tunable } \\
\text { diode-pumped ultrafast Ti:sapphire laser. } \\
\text { Laser experiments include two different mode-locking approaches: one } \\
\text { using a semiconductor saturable absorber mirror (SESAM) and one } \\
\text { using Kerr-lens mode-locking. The experimental setup was optimised } \\
\text { for SESAM mode-locking by ensuring the cavity operated in the middle } \\
\text { of stability zone II, thus creating a second cavity mode waist on the } \\
\text { SESAM. } \\
\text { The same configuration was used for KLM by replacing the SESAM } \\
\text { with a high reflector mirror and moving to the edge of cavity stability. } \\
\text { KLM was then initiated by translating one of the folding mirrors towards } \\
\text { the crystal. }\end{array}$} \\
\hline Pump setup & Pump power & ML mechanism & Self-starting & Output Power & Pulse Duration & Tunability & Reference & \\
\hline $2 \times 452 \mathrm{~nm}$ & $2 \times 1 \mathrm{~W}$ & SESAM & Yes & $101 \mathrm{~mW}$ & $111 \mathrm{fs}$ & No & [1] & \\
\hline $2 \times 520 \mathrm{~nm}$ & $\begin{array}{c}2 \times 1 \mathrm{~W} \\
2 \times 1.5 \mathrm{~W}\end{array}$ & $\begin{array}{c}\text { SESAM } \\
\text { KLM }\end{array}$ & $\begin{array}{l}\text { Yes } \\
\text { No }\end{array}$ & $\begin{array}{l}200 \mathrm{~mW} \\
450 \mathrm{~mW}\end{array}$ & $\begin{array}{l}68 \mathrm{fs} \\
39 \mathrm{fs}\end{array}$ & No & [2] & \\
\hline $2 \times 450 \mathrm{~nm}$ & $2 \times 2.9 \mathrm{~W}$ & SESAM & Yes & $460 \mathrm{~mW}$ & $65 \mathrm{fs}$ & No & {$[3]$} & \\
\hline $2 \times 450 \mathrm{~nm}$ & $2 \times 3.5 W$ & $\begin{array}{l}\text { SESAM } \\
\text { KLM }\end{array}$ & $\begin{array}{l}\text { Yes } \\
\text { No }\end{array}$ & $\begin{array}{l}430 \mathrm{~mW} \\
158 \mathrm{~mW}\end{array}$ & $\begin{array}{l}85 \mathrm{fs} \\
38 \mathrm{fs}\end{array}$ & $\begin{array}{c}50 \mathrm{~nm} \\
120 \mathrm{~nm}\end{array}$ & This work & \\
\hline
\end{tabular}

\section{Experimental setup}
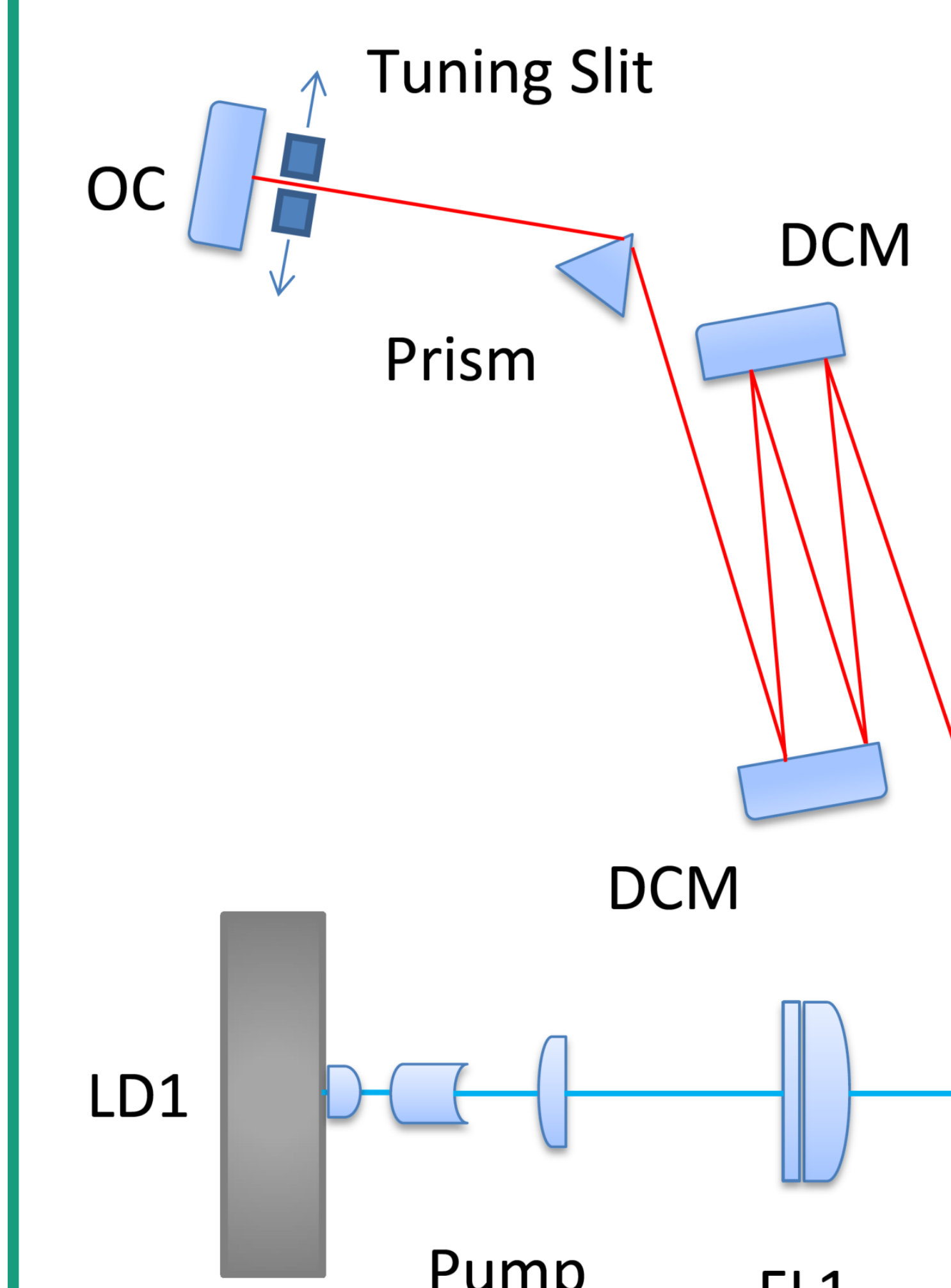

Pump

Focusing

Optics

FL1

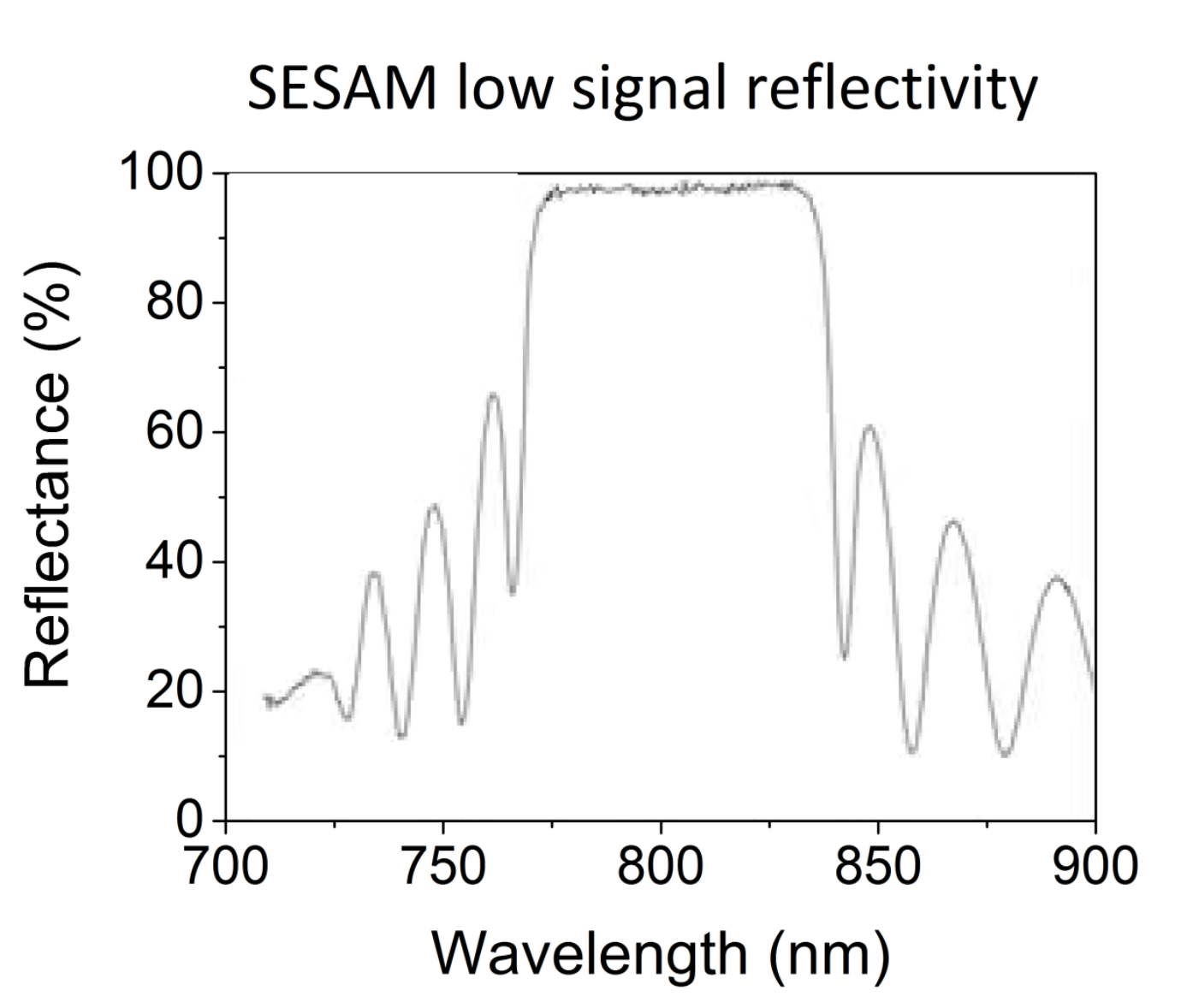

SESAM/HR

mirror
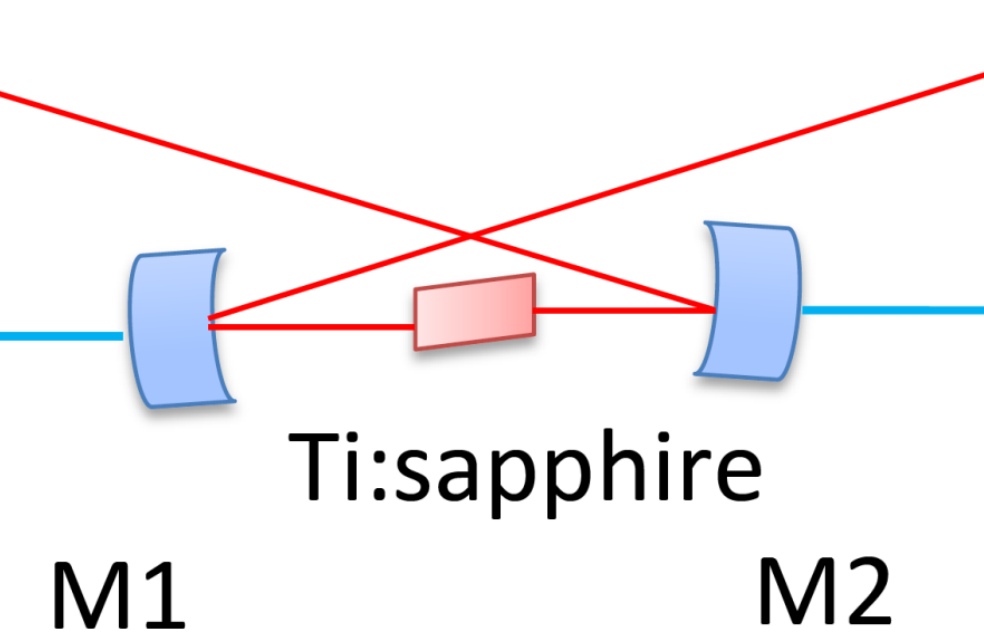

M1

Focusing

Optics
Laser experiments were performed with a Brewster-cut $4.8 \mathrm{~mm}$ long, $3 \mathrm{~mm} \times 3 \mathrm{~mm}$ aperture Ti:sapphire crystal, FOM $>200$, Single pass absorption at $450 \mathrm{~nm}=64 \%, \alpha_{450 \mathrm{~nm}}=2.13 \mathrm{~cm}^{-1}$.

LD1 and LD2: $3.5 \mathrm{~W}, 450 \mathrm{~nm}, 28 \times 1 \mu \mathrm{m}$ emitting area

Pump Focusing Optics: aspheric lens $(f=4.51 \mathrm{~mm})$, concave cylindrical lens $(f=-9.7 \mathrm{~mm})$, convex cylindrical lens $(f=80 \mathrm{~mm})$.

FL1: achromatic doublet lens $(f=100 \mathrm{~mm})$.

FL2: achromatic doublet lens $(f=75 \mathrm{~mm})$.

M1 and M2: dichroic high-reflecting folding mirrors, $75 \mathrm{~mm} \mathrm{ROC.}$

DCM: double chirped mirror $-120 \mathrm{fs}^{2}$ per bounce, $\mathrm{GDD}=-960 \mathrm{fs}^{2}$

Prism: fused silica prism pair, tip-to-tip separation $=50 \mathrm{~cm}, 4 \mathrm{~mm}$ insertion, GDD $=-677 \mathrm{fs}^{2}$. Net cavity GDD: 556 fs$^{2}$ (crystal) - $960 \mathrm{fs}^{2}$ (DCM) - $667 \mathrm{fs}^{2}$ (prism pair) $=-1087 \mathrm{fs}^{2}$

OC: output coupler, $2 \%$ and $5 \%$ for SESAM setup, $1 \%$ for the KLM setup.

SESAM: distributed Bragg reflector structure with a GaAs quantum well, low-signal absorption was $\sim 97.5 \%$ over the range of $775-840 \mathrm{~nm}$, and non-saturable losses are $<1 \%$.

Pump beam waist radii: LD1: $39 \times 13 \mu \mathrm{m}$, and LD2: $30 \times 11 \mu \mathrm{m}$

Laser cavity mode radii in the crystal: $29 \times 16 \mu \mathrm{m}$.

Laser cavity mode radius on the SESAM: $85 \mu \mathrm{m}$.

\section{SESAM mode-locking results}

Input - output characteristics

Tuning range

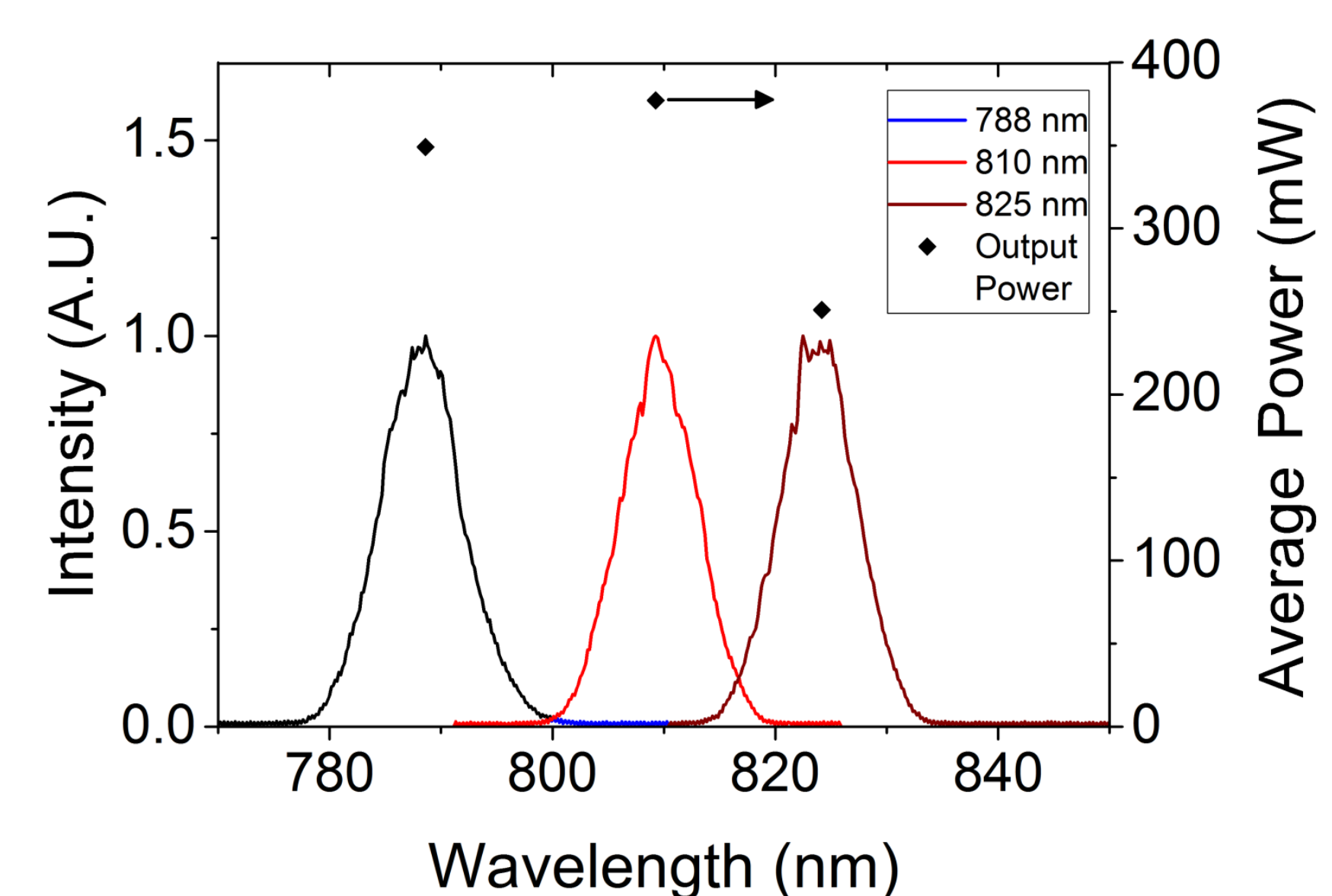

Optical spectrum

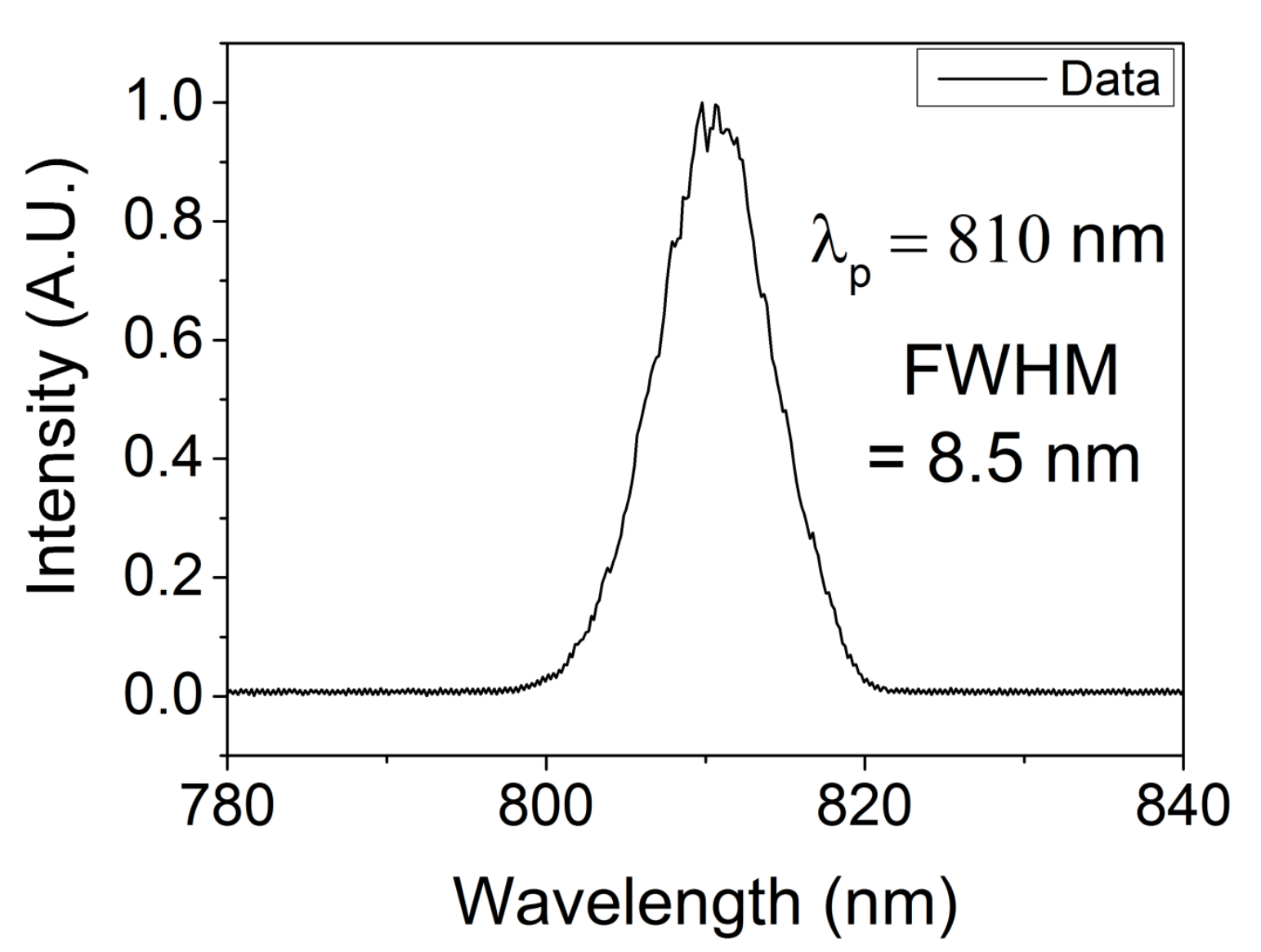

Corresponding autocorrelation

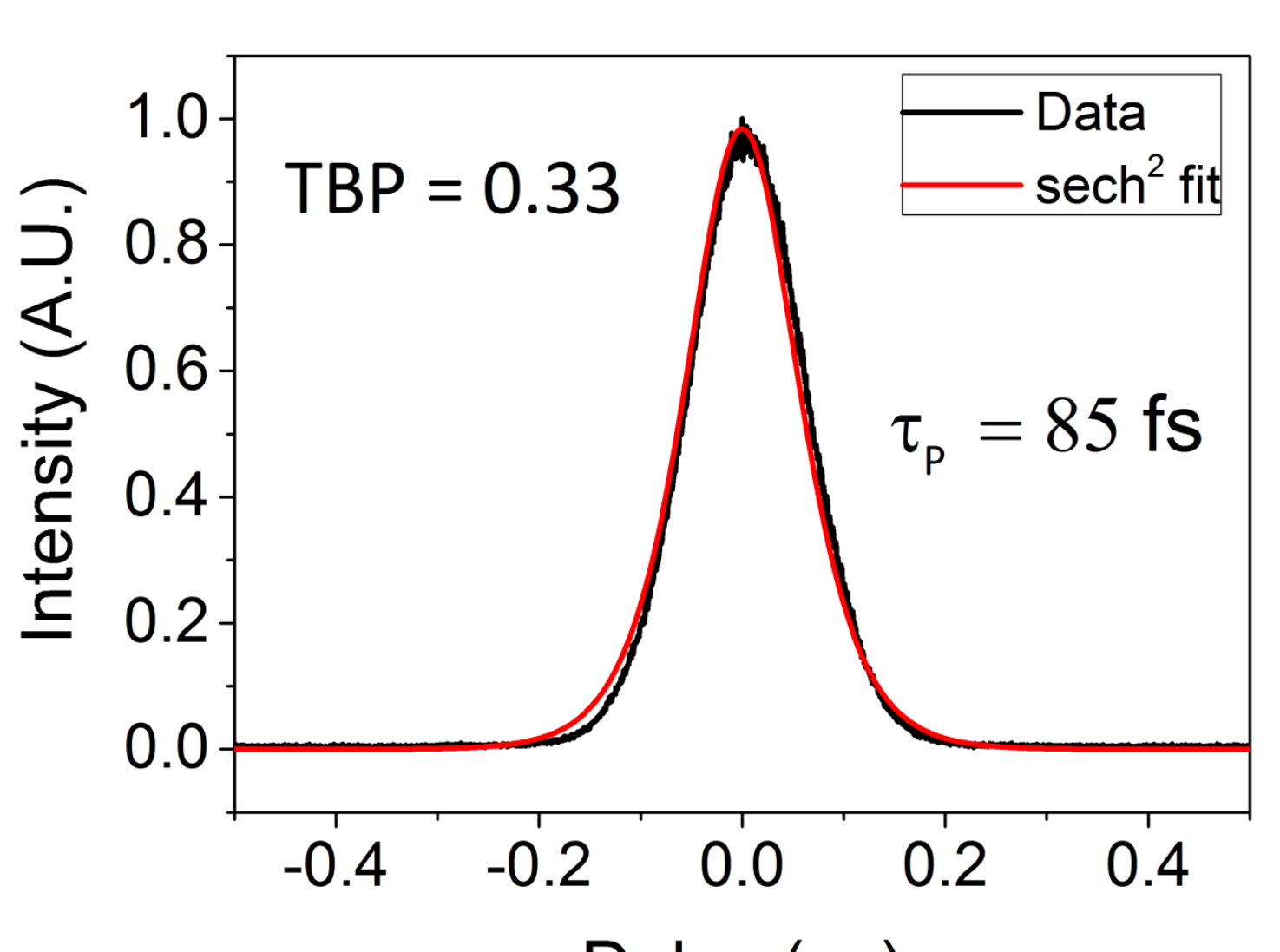

Delay (ps)
RF spectrum

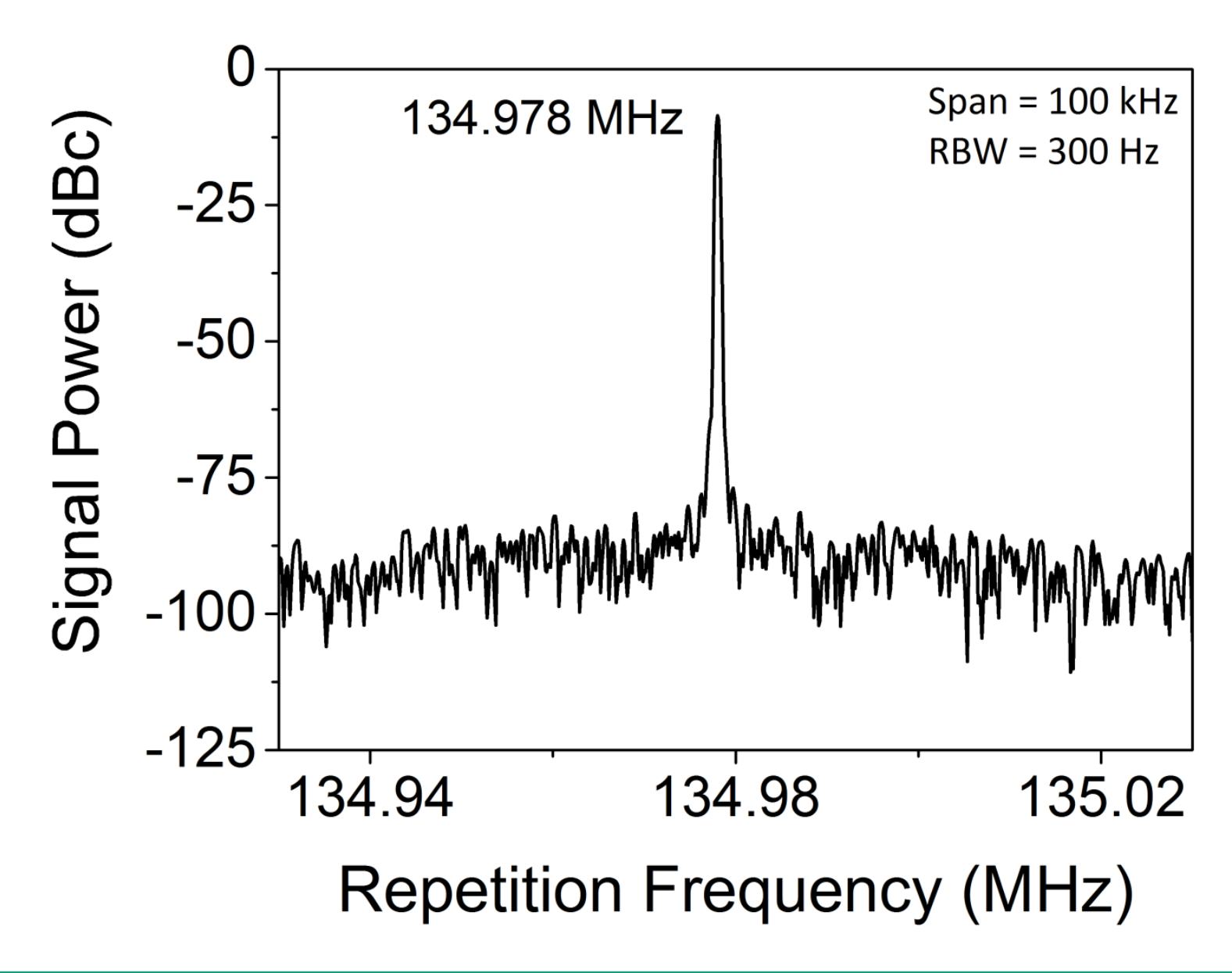

KLM results

Input - output characteristics

Tuning range

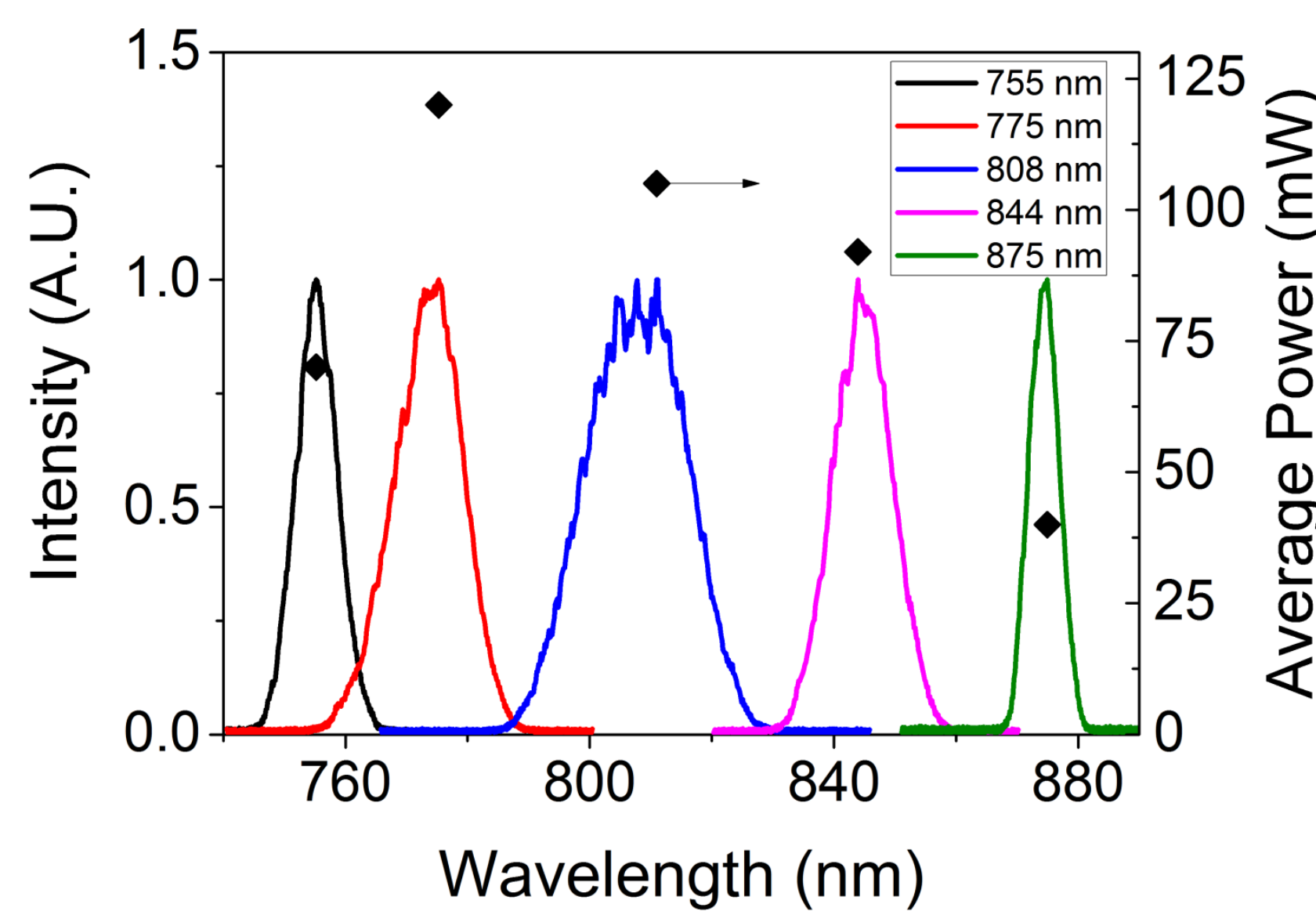

Optical spectrum

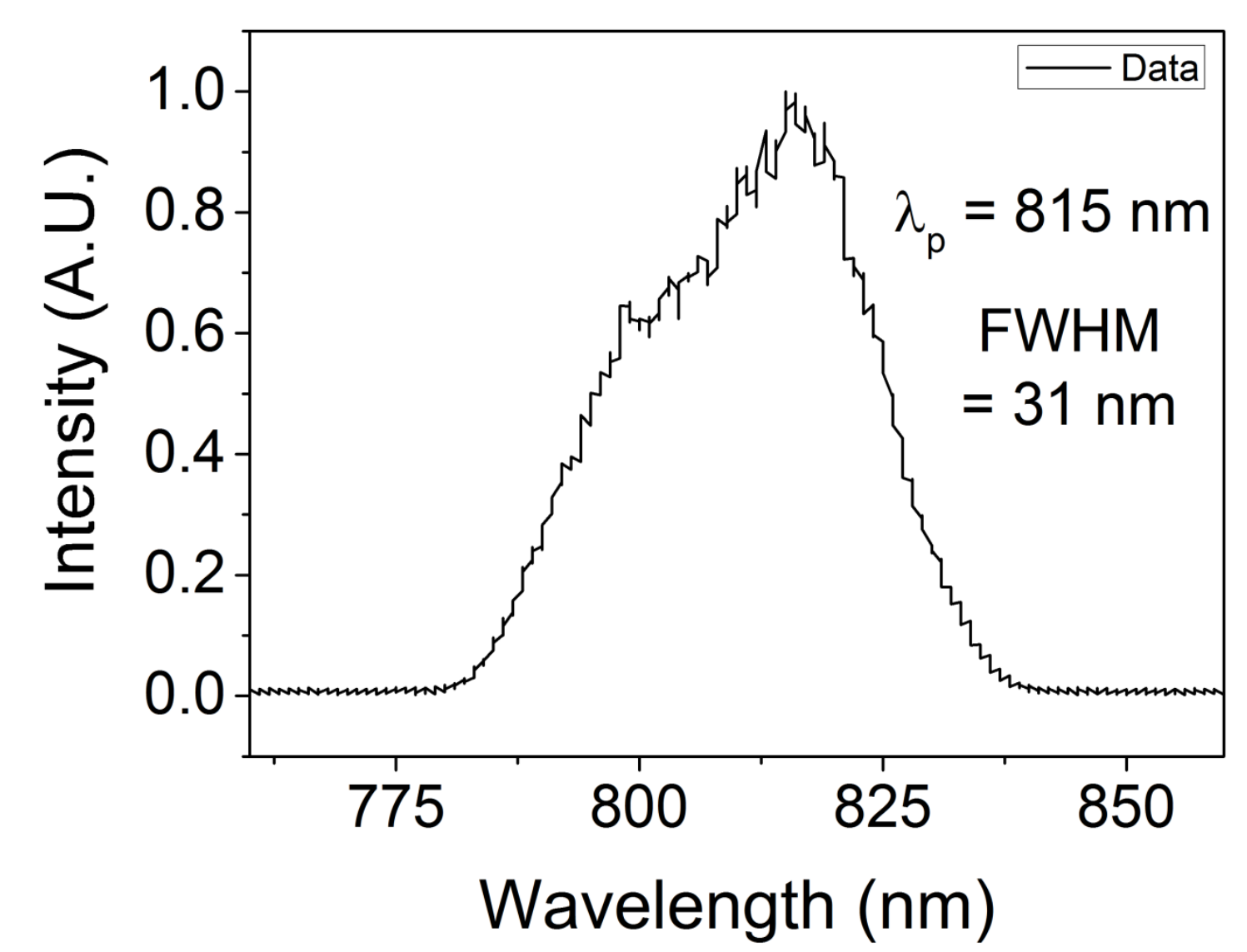

Corresponding autocorrelation

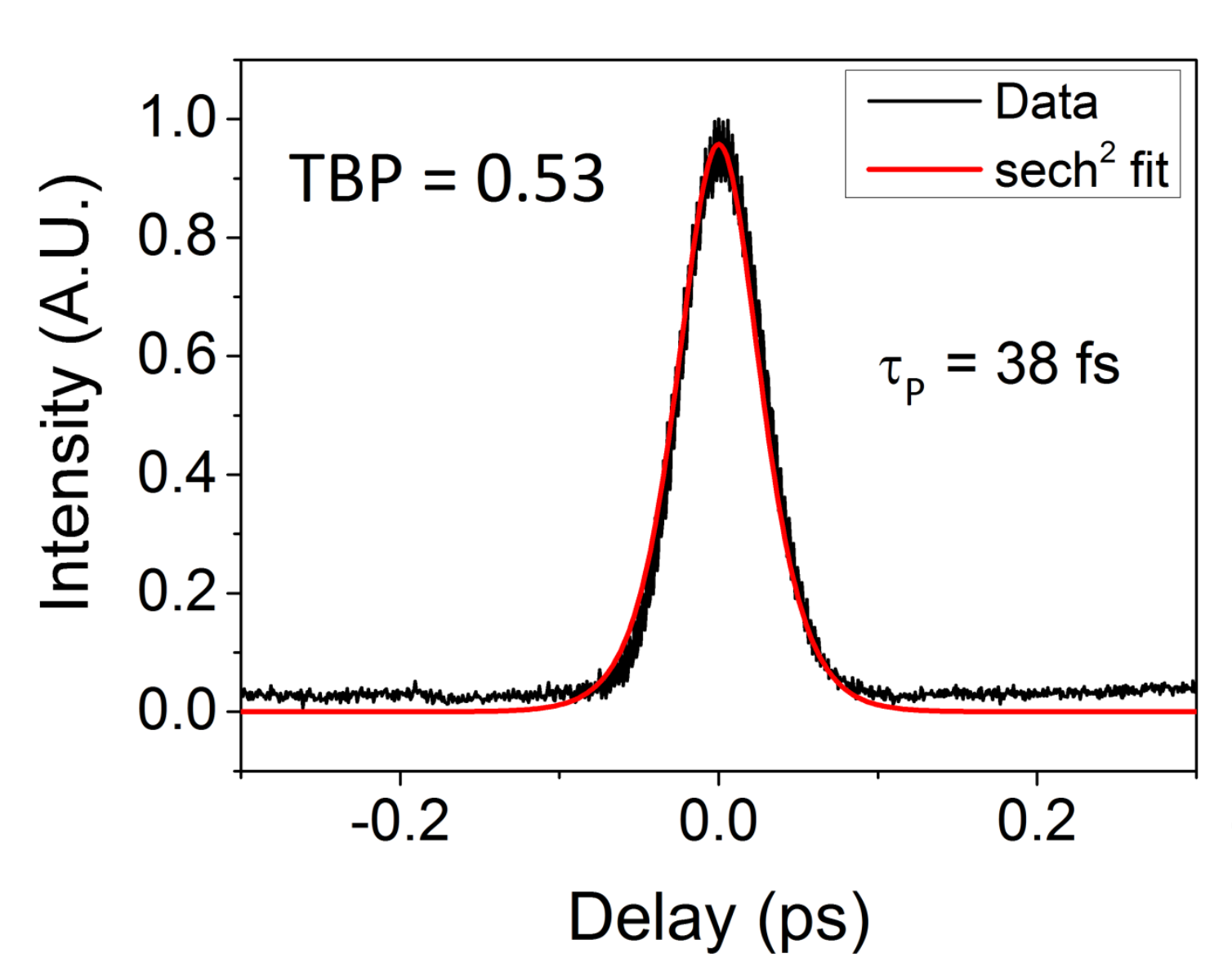

RF spectrum

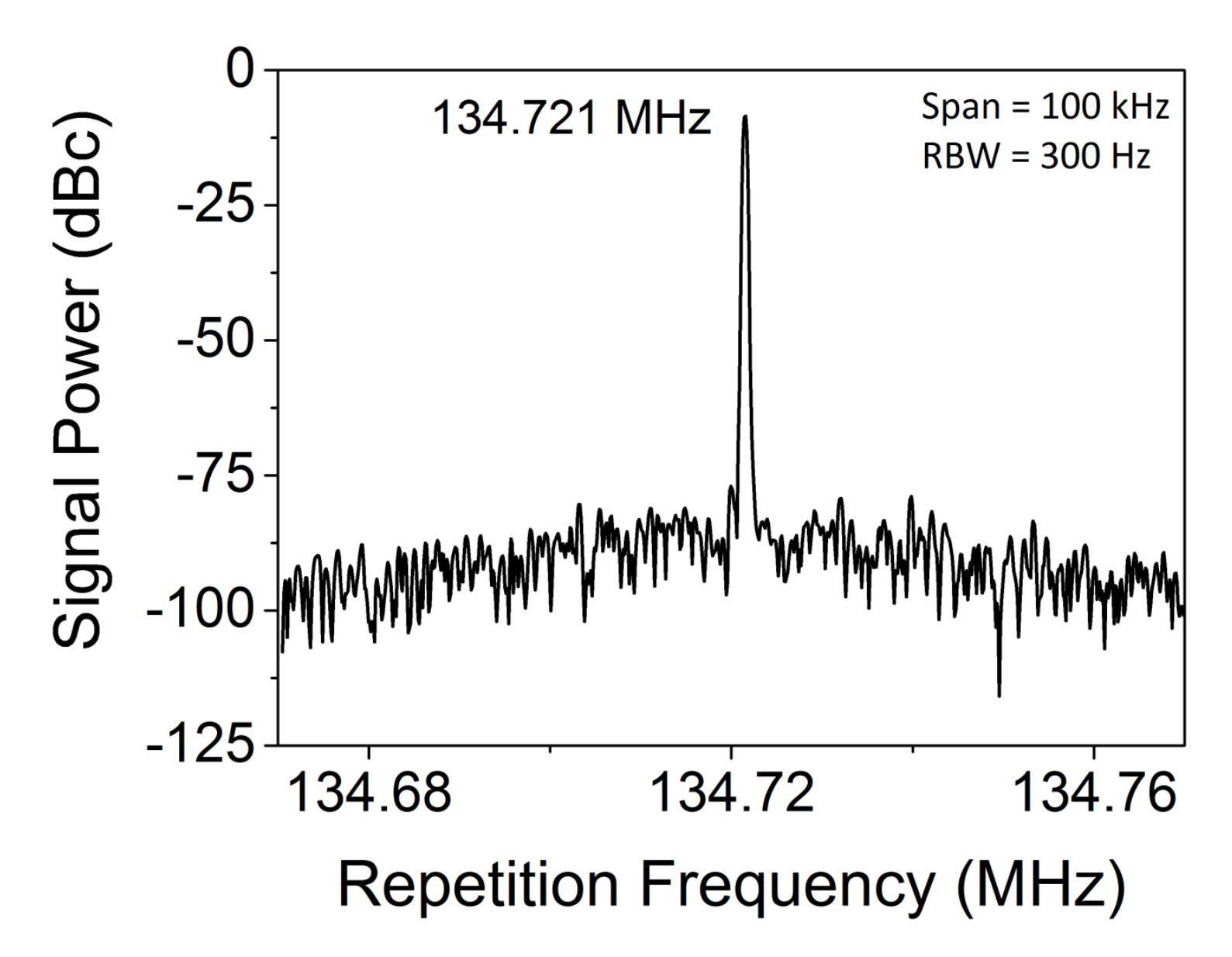

\section{Conclusion}

In conclusion, we have demonstrated average output power as high as $430 \mathrm{~mW}$, pulses as short as 85 fs and tunability as wide as $50 \mathrm{~nm}(2 \% \mathrm{OC})$ for the SESAM mode-locked Ti:sapphire laser. When operated in a KLM regime we were able to achieve a tuning range up to $120 \mathrm{~nm}$, and output power as high as $158 \mathrm{~mW}$. In the case of the SESAM setup tunability was limited by the SESAM reflectivity range, rather than the gain bandwidth of Ti:sapphire. For the KLM configuration, work is underway to further optimise the current setup. Alternatively, wide bandwidth novel saturable absorber materials, such as graphene could be used as a mode-locking element.

\section{References}

1. P. W. Roth, D. Burns, and A. J. Kemp, "Power scaling of a directly diode-laser-pumped Ti:sapphire laser," Opt. Express 20, 20629-20634 K. Gürel, V. J. Wittwer, M. Hoffmann, C. J. Saraceno, S. Hakobyan, B. Resan, A. Rohrbacher, K. Weingarten, S. Schilt, and T. Südmeyer, "Green-diode-pumped femtosecond Ti:Sapphire laser with up to $450 \mathrm{~mW}$ average power," Opt. Express 23, 30043-30048 (2015). A. Ron-hroacher, O. E. Olarte, V. Villamaina, P. Loza-Alvarez, and B. Resan, "Multiphoton imaging with blue-diode-pumped SESAM-modelocked
Ti:sapphire oscillator generating $5 \mathrm{~nJ} 82$ ts pulses," Opt. Express 25, $10677-10684$ (2017).

Acknowledgements: This work was funded by UK Engineering and Physical Sciences Research Council (EPSRC), via the Centre for Doctoral Training in Applied Photonics and Fraunhofer UK Research Limited. AJK acknowledges support from the Royal Academy of
Engineering. 